\title{
Estableciendo la unidad de atención a la accesibilidad digital en el Tecnológico Nacional de México / IT Aguascalientes
}

\section{Establishing the digital accessibility care unit at the Tecnológico Nacional de México / IT Aguascalientes}

\author{
Ricardo Mendoza-González \\ Tecnológico Nacional de México / IT Aguascalientes, Aguascalientes, México \\ mendozagric@aguascalientes.tecnm.mx \\ https://orcid.org/0000-0002-8934-8067 \\ Juan-Miguel Tovar-Luevano \\ Tecnológico Nacional de México / IT Aguascalientes, Aguascalientes, México \\ admon aguascalientes@tecnm.mx \\ https://orcid.org/0000-0001-5197-3097 \\ Rafael Portillo-Rosales \\ Tecnológico Nacional de México / IT Aguascalientes, Aguascalientes, México \\ rafael.pr@aguascalientes.tecnm.mx \\ https://orcid.org/0000-0001-6610-9533
}

(Recibido: 27/10/2021; Aceptado: 15/11/2021; Versión final recibida: 15/12/2021)

Cita del artículo: Mendoza-González, R., Tovar-Luevano, J.M. y Portillo-Rosales, R. (2022). Estableciendo la unidad de atención a la accesibilidad digital en el Tecnológico Nacional de México / IT Aguascalientes. Revista Cátedra, 5(1), 106-118.

\section{Resumen}

El objetivo de este trabajo fue presentar el conjunto de servicios básicos de la Unidad de Atención a la Accesibilidad Digital del Tecnológico Nacional de México / IT Aguascalientes (TecNM/ITA), así como su integración inicial a la estructura orgánica de la institución. Dichas acciones se llevaron a cabo en el marco del proyecto "Asistencia tecnológica a la accesibilidad en la Educación Superior Virtual, EduTech", número de registro: 609785-EPP1-2019-1-ES-EPPKA2-CBHE-JP, cofinanciado por el programa ERASMUS+ de la Unión

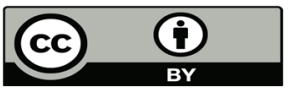


Europea. En este sentido, se enfatizó la implementación de los resultados derivados de dos entregables de dicho proyecto: "1.1 Informe del estado de arte de unidades de atención a la accesibilidad tecnológica aplicada a la Educación Superior", y "2.1 Guía de implantación y procedimientos de gestión de una unidad de atención a la accesibilidad tecnológica aplicada a la Educación Superior". Los hallazgos y recomendaciones de estos entregables permitieron establecer los cimientos de la primera Unidad de Atención a la Accesibilidad Digital en todo el sistema nacional de tecnológicos, el cual cuenta con 254 institutos a lo largo del país. Se espera que el contenido de este artículo inspire a otras Instituciones de Educación Superior (IES) para que conformen sus propias Unidad de Atención a la Accesibilidad Digital.

\section{Palabras clave}

Accesibilidad digital o tecnológica, adaptaciones curriculares, adaptaciones razonables, IES, unidades de atención.

\section{Abstract}

The objective of this work was to present the set of basic services of the Digital Accessibility Attention Unit of the Tecnológico Nacional de México / IT Aguascalientes (TecNM/ITA), as well as its initial integration into the organizational structure of the institution. These actions were carried out within the framework of the project "Technological assistance to accessibility in Virtual Higher Education, EduTech", registration number: 609785-EPP-12019-1-ES-EPPKA2-CBHE-JP, co-financed by the ERASMUS+ program of the European Union. In this sense, emphasis was placed on the implementation of the results derived from two deliverables of said project: "1.1 Report on the state of the art of technological accessibility care units applied to Higher Education", and "2.1 Guide for the implementation and management procedures of a technological accessibility care unit applied to Higher Education". The findings and recommendations of these deliverables made it possible to establish the foundations of the first Digital Accessibility Attention Unit in the entire national technological system, which has 254 institutes throughout the country. It is hoped that the content of this article will inspire other Higher Education Institutions (HEI) to create their own Digital Accessibility Attention Units.

\section{Keywords}

Attention units, curricular adjustments, digital or technological accessibility, HEI, reasonable adjustments.

\section{Introducción}

Para proporcionar una idea más clara del contexto de este artículo, se describirán en esta sección tres aspectos importantes: los objetivos y características de las Unidades de Atención a la Accesibilidad Digital, un resumen del proyecto "Asistencia tecnológica a la accesibilidad en la Educación Superior Virtual, EduTech", y un resumen del sistema nacional de tecnológicos en México.

\subsection{Unidades de Atención a la Accesibilidad Tecnológica}

Los Servicios de apoyo a Personas con Discapacidad en Universidades también son conocidos como: Programa/Servicio/Unidad/Oficina de Atención/Apoyo a la Discapacidad, Unidad de Atención a la Accesibilidad Tecnológica/Digital, Unidad para la Igualdad y Atención a la Discapacidad, Unidad de Educación Inclusiva, Centro de apoyo al estudiante, entre otros nombres similares. El objetivo general de este tipo de servicios es "garantizar la plena inclusión y participación del estudiantado universitario con discapacidad, contemplando su individualidad, a través de una efectiva igualdad de oportunidades y no 
discriminación en la vida académica, así como la promoción de la sensibilización y concienciación de todos los miembros de la comunidad" (Fundación Universia, 2017, p. 23). El logro de este objetivo conlleva los siguientes aspectos específicos: incorporar accesibilidad al proceso de ingreso a la universidad; sensibilizar y concientizar a la comunidad universitaria para la adecuada implementación de las políticas y normas de accesibilidad en favor de las personas con discapacidad; ofrecer servicios de apoyo académico a los estudiantes con discapacidad; asegurar la accesibilidad en el ambiente universitario (infraestructura, equipamiento, información, servicios, proceso de enseñanzaaprendizaje) incluyendo el espacio virtual; fomentar la colaboración y la sinergia entre la comunidad universitaria y fundaciones, organizaciones, o instituciones externas en favor de la atención a los estudiantes con discapacidad; modificar los lineamientos actuales (y/o generar nuevos) con el fin de formalizar y preservar la adecuada atención a las necesidades de los estudiantes con discapacidad; fomentar en la industria y el gobierno la inserción laboral y la futura contratación de estudiantes / egresados con discapacidad; incorporar programas de formación en accesibilidad a los involucrados en el proceso de enseñanzaaprendizaje; y modernizar los procesos de atención en los centros universitarios de servicio mediante el fomento de una cultura inclusiva y en favor de las personas con discapacidad (Fundación Universia, 2017; Peralta y Comunitaril, 2011; Porto Castro y Gerpe-Pérez, 2020; United Nations, 2006).

Con base en dichos objetivos específicos y en los aspectos generales a considerar en este tipo de servicios puntualizados por Fundación Universia (2018), se identificaron las siguientes características ideales para los servicios de atención a la discapacidad en las IES:

- La unidad opera directamente como un servicio, oficina, o área interna/específica en la universidad. Aunque esta es la forma de operación más común, también se puede operar a través de una fundación, o bien como una tarea compartida entre 2 o más servicios, oficinas, o áreas internas en la universidad.

- La unidad depende orgánicamente de una vicerrectoría o subdirección (estructura orgánica más común), gerencia u otra área administrativa, o de una fundación.

- Los equipos de trabajo de la unidad se integran de manera multidisciplinaria (psicólogos, pedagogos y psicopedagogos, fisioterapeutas, intérpretes de Lengua de Signos, entre otros) e incluyente (por ejemplo, hombres, mujeres, personal con discapacidad).

- La unidad de atención de una universidad trabaja en conjunto con sus similares en otras universidades, otras unidades de atención en la propia universidad (por ejemplo, atención psicológica), Instituciones públicas y/o privadas que trabajan con discapacidad.

- La unidad de atención fomenta la participación voluntaria de estudiantes reconociendo con créditos académicos su colaboración en los servicios de apoyo a las personas con discapacidad en la universidad.

- La unidad de atención cuenta con un proceso de mejora continua o un sistema interno de evaluación, propio o basado en alguna norma de aseguramiento de la calidad (por ejemplo, norma UNE-EN ISO 9001:2015).

- La unidad considera estrategias personalizadas de inducción al entorno universitario, orientación, tutoría, seguimiento, apoyo, y asesoramiento para estudiantes con discapacidad, involucrando los procesos de ingreso/inscripción de nuevos estudiantes, de formación universitaria, y de egreso, e incluso la inserción laboral.

- La unidad se asegura de mantener el rigor académico en la formación de estudiantes con discapacidad manteniendo una constante comunicación con estudiantes y profesores para resolver problemas de accesibilidad en las dependencias, la información o las adaptaciones curriculares necesarias.

\section{Licencia Creative Commons Atribución 4.0 Internacional (CC BY 4.0)}

Revista Cátedra, 5(1), pp. 106-118, enero-junio 2022. e-ISSN:2631-2875

https://doi.org/10.29166/catedra.v5i1.3293 
- La unidad asegura mecanismos para que los estudiantes con discapacidad participen en los diversos programas de movilidad estudiantil (estatal, nacional, internacional) incluyendo la participación de estudiantes con la industria (por ejemplo, estadías/prácticas).

- La unidad cuenta con los mecanismos necesarios para el fomento de la empleabilidad e inclusión laboral de estudiantes con discapacidad mediante estrategias como la orientación laboral y acompañamiento en la definición de objetivos profesionales. También se llevan a cabo programas de prácticas en empresas, comunicación de ofertas laborales recibidas e incluso la gestión de bolsas de trabajo a través de las áreas de vinculación universitaria y/o servicio de empleo de la universidad.

- La unidad asegura la adaptación de puestos de estudio para personas con discapacidad en el contexto de los conceptos de adaptaciones curriculares y adaptaciones razonables, por ejemplo, Reserva de asientos en las aulas, grabación de clases, adaptación de mobiliario, incorporación de bucles magnéticos en salones de actos, materiales educativos accesibles, ampliación de tiempo para exámenes, adaptación curricular, entre otros.

- La unidad asegura las adaptaciones razonables pudiéndose realizar de diferentes maneras de acuerdo con los recursos disponibles en la universidad, por ejemplo, dotación de software y hardware específico, la digitalización de contenidos con criterios de accesibilidad, y el subtitulado de material audiovisual.

\subsection{El proyecto "Asistencia tecnológica a la accesibilidad en la Educación Superior Virtual, EduTech"}

La idea del proyecto fue surge en la Universidad de Alcalá de Henares, Madrid, España, como iniciativa del Dr. Salvador Otón Tortosa, Profesor investigador de dicha universidad. El proyecto fue seleccionado para ser co-financiado por el programa ERASMUS+, "Acción clave 2: Desarrollo de Capacidades en el Ámbito de la Educación Superior", por sus siglas en inglés: "KA2: CBHE", de la Unión Europea en su convocatoria de 2019. El proyecto EduTech busca generar unidades de atención para gestionar los diferentes aspectos relacionados con la accesibilidad tecnológica en la educación superior, propósito que se integra desde el mismo acrónimo del proyecto: "Edu" que representa a la educación inclusiva y "Tech" que representa las Tecnologías de la Información y la Comunicación (TIC) en las IES, y en Náhuatl (lengua indígena mexicana) representa los términos "Nosotros o para nosotros".

El proyecto estará vigente hasta el año 2023 y su desarrollo se lleva a cabo mediante el trabajo colaborativo de ocho instituciones socias: la Universidad Alcalá (UAH, España), Østfold University College (HioF, Noruega), Universidad de Alicante (UA, España), Universidade Aberta (UAb, Portugal), Tecnológico Nacional de México/Instituto Tecnológico de Aguascalientes (TecNM / ITA, México), Universidad Veracruzana (UV, México), Universidad Politécnica Salesiana (UPS, Ecuador), y Universidad del Azuay (UDA, Ecuador).

El proyecto EduTech se enfoca en plantear soluciones a cinco problemáticas principales en el contexto de la accesibilidad tecnológica en la educación superior:

1. La carencia de un modelo de unidad o departamento en las IES que atienden los diferentes aspectos relacionados con la accesibilidad tecnológica.

2. La falta de documentación o material formativo para las IES en relación a casos de éxito y buenas prácticas en: Atención de estudiantes en aspectos de tecnología accesible; Herramientas para la formación virtual accesible; Campus virtuales accesibles con características de accesibilidad; Objetos de aprendizaje y OERs accesibles; Cursos Masivos Abiertos Online (MOOC) accesibles; Calidad en la

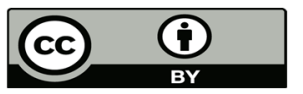

Licencia Creative Commons Atribución 4.0 Internacional (CC BY 4.0)

Revista Cátedra, 5(1), pp. 106-118, enero-junio 2022. e-ISSN: 2631-2875

https://doi.org/10.29166/catedra.v5i1.3293 
formación virtual accesible y Aspectos de formación a involucrados en el proceso de enseñanza-aprendizaje.

3. La necesidad de mejorar la infraestructura tecnológica y capacidades de gestión para una Educación Superior Virtual Accesible en las universidades socias latinoamericanas.

4. La carencia de programas de formación masivos en temas de la "Agenda de nuevas cualificaciones y empleos" de la Estrategia 2020, orientados especialmente para grupos vulnerables como las personas con discapacidad.

5. La falta de un espacio común de unidades de accesibilidad tecnológica en las IES, para compartir casos de éxitos y buenas prácticas.

Para lograr lo anterior, se establecieron los siguientes objetivos específicos de la acción.

1. Ayudar a las Instituciones de Educación Superior (IES) de América Latina (AL) y europeas en la instauración de unidades de atención tecnológicas de la accesibilidad, replicables y adaptables según las características de las instituciones, y formar al personal implicado en dichas unidades de atención.

2. Crear un volumen de accesibilidad, conformado con seis guías acerca de los principales temas de accesibilidad: (1) Guía para la implementación de unidades de gestión de la accesibilidad tecnológica en las IES; (2) Guía técnica para campus virtuales accesibles y adaptativos para la implementación de e-learning; (3) Guía técnica para la creación y gestión de OERs y MOOCs accesibles; (4) Guía para autoevaluación de la calidad virtual accesible; (5) Guía de formación en accesibilidad del personal involucrado en el proceso de enseñanza aprendizaje. (6) Guía sobre buenas prácticas para la inserción laboral de personas con discapacidad.

3. Desarrollar una serie de herramientas de apoyo a la accesibilidad para docentes y estudiantes (por ejemplo: plugins de accesibilidad para Moodle, repositorios de objetos de aprendizaje y OERs, y simuladores laborales) implementando los resultados y recomendaciones de las guías confeccionadas en el objetivo 2. Se desarrollarán.

4. Mejorar la infraestructura tecnológica y capacidades de gestión de las IES socias para una Educación Superior Virtual Accesible.

5. Fomentar la inserción laboral y educativa de estudiantes con discapacidad.

6. Apoyar a las IES latinoamericanas socias para avanzar en la creación de un espacio de educación superior virtual inclusiva, como forma de consolidar el espacio común ALCUE (Cumbre América Latina, el Caribe y la Unión Europea) de Educación Superior, mediante la expansión de redes de cooperación existentes con una red de cooperación de unidades de atención a la accesibilidad tecnológica.

\subsection{El sistema nacional de tecnológicos y el Tecnológico Nacional de México}

Los primeros Institutos Tecnológicos (IT) surgieron en México en el año de 1948, fueron Durango y Chihuahua los estados en los que se asentaron, con el propósito de impulsar la ciencia y tecnología regional. Poco tiempo después se fundaron los de Saltillo (1951) y Ciudad Madero (1954). Hacia 1955, estos primeros cuatro Tecnológicos atendían una población escolar de 1,795 alumnos, de los cuales 1,688 eran hombres y sólo 107 mujeres. En 1957 inició operaciones el IT de Orizaba. En 1959, los Institutos Tecnológicos son desincorporados del Instituto Politécnico Nacional, para depender, por medio de la Dirección General de Enseñanzas Tecnológicas Industriales y Comerciales, directamente de la Secretaría de Educación Pública. En el libro La Educación Técnica en México. Institutos Tecnológicos Regionales, editado por la Secretaría de Educación Pública en 1958, se marcó la desincorporación plena de los IT y el inicio de una nueva etapa caracterizada por la respuesta que dan estas instituciones a las necesidades propias del medio geográfico y social, y al desarrollo industrial de la zona en que se ubican. Al cumplirse los primeros veinte 
años, ya se contaba con diecisiete IT, los cuales estaban presentes en catorce estados de la República Mexicana. En la década siguiente (1968-1978), se fundaron otros 31 Tecnológicos, para llegar a un total de 48 planteles distribuidos en veintiocho entidades del país. Durante esta década se crearon también los primeros centros de investigación y apoyo a la educación tecnológica, es decir, el Centro Interdisciplinario de Investigación y Docencia en Educación Tecnológica (CIIDET, 1976) en Querétaro y el Centro Regional de Optimización y Desarrollo de Equipo (CRODE), en Celaya. En 1979 se constituyó el Consejo Nacional del Sistema Nacional de Educación Técnica (COSNET), el cual representó un nuevo panorama de organización, surgiendo el Sistema Nacional de Educación Tecnológica, siendo los Institutos Tecnológicos parte fundamental para la integración del Sistema Nacional de Institutos Tecnológicos (SNIT). De 1978 a 1988 se fundaron doce nuevos Tecnológicos, dos Centros Regionales de Optimización y Desarrollo de Equipo (Chihuahua y Mérida), y el Centro Nacional de Investigación y Desarrollo Tecnológico (CENIDET).

La investigación y los posgrados se impulsaron con gran intensidad gracias a la creación progresiva de los Centros Regionales de Estudios de Graduados e Investigación Tecnológica (CREGIT) en cada uno de los planteles. Para 1988, los IT atendían una población escolar de 98,310 alumnos, misma que en los cinco años siguientes creciera hasta 145,299 , con una planta docente de 11,229 profesionales y 7,497 empleados como personal de apoyo y asistencia a la educación. En 1990 iniciaron actividades los Institutos Tecnológicos Descentralizados, con esquemas distintos a los que operaban en los IT Federales, ya que se crearon como organismos descentralizados de los Gobiernos Estatales. En 1993 se crea el CRODE de Orizaba. Mientras que en 2005 se reestructuró el Sistema Educativo Nacional por niveles, lo que trajo como resultado la integración de los Institutos Tecnológicos a la Subsecretaría de Educación Superior (SES), transformando a la Dirección General de Institutos Tecnológicos (DGIT) en Dirección General de Educación Superior Tecnológica (DGEST). Como consecuencia de esta reestructuración, se desincorpora el nivel superior de la Dirección General de Ciencia y Tecnología del Mar y de la Dirección General de Educación Tecnológica Agropecuaria y se incorpora a la recién creada DGEST. El 23 de julio de 2014, fue publicado en el Diario Oficial de la Federación, el Decreto Presidencial por el que se crea la institución de educación superior tecnológica más grande de nuestro país, el Tecnológico Nacional de México (TecNM). De acuerdo con el Decreto citado, el TecNM se funda como un órgano desconcentrado de la Secretaría de Educación Pública, que sustituye a la unidad administrativa que se hacía cargo de coordinar este importante subsistema de educación superior. El Tecnológico Nacional de México está constituido por 254 instituciones, de las cuales 126 son Institutos Tecnológicos Federales, 122 Institutos Tecnológicos Descentralizados, cuatro Centros Regionales de Optimización y Desarrollo de Equipo (CRODE), un Centro Interdisciplinario de Investigación y Docencia en Educación Técnica (CIIDET) y un Centro Nacional de Investigación y Desarrollo Tecnológico (CENIDET). En estas instituciones, el TecNM atiende a una población escolar de más de 600 mil estudiantes en licenciatura y posgrado en todo el territorio nacional, incluida la Ciudad de México (TecNM, 2021).

\subsection{Planteamiento del problema}

A pesar de que el sistema de tecnológicos en México tiene más de 70 años en operación, fue hasta 2018 que se iniciaron trabajos específicamente orientados a la accesibilidad y la inclusión tecnológica, esto mediante un diplomado de educación inclusiva. Por lo tanto, es necesario establecer estrategias que contribuyan a fortalecer las alternativas actuales en favor de los estudiantes con discapacidad en la institución. En este sentido, el modelo de Unidad de Atención a la Accesibilidad Digital es nuevo en el sistema, requiriéndose de información fundamentada, orientación, y capacitación para iniciar un adecuado desarrollo de esta estrategia.

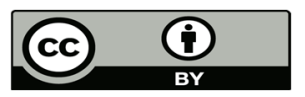

Licencia Creative Commons Atribución 4.0 Internacional (CC BY 4.0)

Revista Cátedra, 5(1), pp. 106-118, enero-junio 2022. e-ISSN: 2631-2875

https://doi.org/10.29166/catedra.v5i1.3293 
La implementación del "1.1 Informe del estado de arte de unidades de atención a la accesibilidad tecnológica aplicada a la Educación Superior" y de la "2.1 Guía de implantación y procedimientos de gestión de una unidad de atención a la accesibilidad tecnológica aplicada a la Educación Superior", así como la co-financiación provista por la Unión Europea a través del proyecto EduTech, ha permitido mitigar dicha situación dándose inicio a la planificación de la Unidad de Atención a la Accesibilidad Tecnológica en el instituto, catalogando al TecNM/ITA como pionero en el establecimiento de este tipo de servicios de apoyo dentro del sistema nacional de tecnológicos.

A través de este artículo se pretende difundir al proyecto EduTech fomentando la implementación de sus entregables una vez que estén disponibles. En este sentido, la sección 2 resume la implementación del "1.1 Informe del estado de arte de unidades de atención a la accesibilidad tecnológica aplicada a la Educación Superior" y de la "2.1 Guía de implantación y procedimientos de gestión de una unidad de atención a la accesibilidad tecnológica aplicada a la Educación Superior" para integrar la unidad de atención a la accesibilidad digital del TecNM/ITA, destacando sus funciones en la subsección 2.1, y su estructura orgánica en la subsección 2.2. Finalmente, la sección 3 presenta las primeras conclusiones obtenidas tras la puesta en práctica de los hallazgos derivados de los entregables mencionados.

\section{Integrando la Unidad de Atención a la Accesibilidad Digital}

El proyecto EduTech hizo posible la integración de la unidad de atención en el instituto no solo mediante el cofinanciamiento del programa ERASMUS+ de la Unión Europea; sino a través de los resultados y recomendaciones de sus entregables "1.1 Informe del estado de arte de unidades de atención a la accesibilidad tecnológica aplicada a la Educación Superior", y "2.1 Guía de implantación y procedimientos de gestión de una unidad de atención a la accesibilidad tecnológica aplicada a la Educación Superior". La conducción esencial de dichos documentos permitió visualizar las funciones de la unidad de atención a la accesibilidad digital, y su integración a la estructura orgánica del instituto. Asimismo, se han propuesto las siguientes declaraciones preliminares para la misión y visión de la unidad.

- Misión, establecer una comunidad accesible donde los estudiantes con discapacidad tengan la misma oportunidad de participar plenamente en todos los aspectos del entorno educativo, promover la independencia de los estudiantes, y asegurar que los estudiantes sean reconocidos por sus habilidades y conocimientos por encima de su discapacidad.

- Visión, un ambiente educativo - incluyendo: Oportunidades y actividades educacionales; académicas; laborales; recreativas; sociales; y deportivas- en el Tecnológico Nacional de México /IT Aguascalientes universalmente accesible para todos los estudiantes sin la necesidad de intervenciones, adaptaciones, y/o diseños especiales gestionados por la unidad de atención a la accesibilidad.

En las siguientes subsecciones se describen las actividades correspondientes resaltando su estatus actual indicándose: en planificación, en desarrollo, o implementadas.

\subsection{Funciones de la Unidad de Atención a la Accesibilidad Digital}

Los fundamentos del estado del arte de unidades de atención a la accesibilidad tecnológica aplicada a la educación superior, y las recomendaciones de la guía de implantación y procedimientos de gestión de una unidad de atención a la accesibilidad tecnológica aplicada a la educación superior, permitieron identificar el siguiente conjunto de servicios de apoyo tecnológico para estudiantes universitarios con discapacidad: 
- Asistencia tecnológica a estudiantes con discapacidad visual (parcial), auditiva (parcial), y de movilidad (EN PLANIFICACIÓN), mediante el equipamiento adquirido con el apoyo de la Comisión Europea a través del proyecto "Asistencia tecnológica a la accesibilidad en la Educación Superior Virtual", se desarrollarán tecnologías de asistencia a la accesibilidad en la educación virtual accesible, incluyendo: campus virtual accesible, recursos educativos abiertos accesibles, plugins accesibles para Moodle, y simuladores laborales en 3D. Asimismo, se contará con quince estaciones de trabajo accesibles, que estarán disponibles para estudiantes con discapacidad visual (parcial), auditiva (parcial), y de movilidad que deseen utilizarlas para realizar actividades académicas de sus clases presenciales. Adicionalmente, se ha presentado a las autoridades del instituto una estrategia basada en el fomento, diseño, desarrollo, e implementación de adaptaciones curriculares (Alcantud, Ávila, y Asensi, 2000; Corredor Ponce, 2016) y adaptaciones razonables (Ortego Hernando, 2000; Sandoval, Morgado, y Doménech, 2020). Esta iniciativa que gestionaría la unidad de atención a la accesibilidad digital involucra la detección de estudiantes con discapacidad durante el proceso de matriculación; la notificación a los profesores que tendrían por alumnos a dichos aspirantes; y la negociación de las adaptaciones curriculares y razonables en función de los recursos disponibles. Se visualiza un servicio de voluntariado estudiantil como apoyo académico a estudiantes con discapacidad basado en el modelo de Victoria University of Wellington ${ }^{1}$ (Nueva Zelanda). Así como el reforzamiento del programa de tutoría académica actual mediante la incorporación de aspectos accesibles para atender de mejor manera a estudiantes con discapacidad.

- Asistencia a los programas de educación a distancia que se imparten en el instituto incorporando aspectos de accesibilidad en la educación virtual (EN PLANIFICACIÓN), la unidad de atención a la accesibilidad digital colaborará con la coordinación de educación a distancia del Tecnológico Nacional de México / IT Aguascalientes para desarrollar elementos tecnológicos accesibles (por ejemplo, plugins para Moodle), e implementarlos en los cursos de los dos programas de pregrado en la modalidad de educación a distancia que se ofertan actualmente.

- Capacitación a los docentes del instituto en aspectos esenciales de la educación virtual accesible (EN PLANIFICACIÓN), a través de la unidad de atención a la accesibilidad digital se gestionará y se llevará a cabo un programa de capacitación accesible inicialmente dirigido a los docentes y posteriormente extendido al personal administrativo. Actualmente, se ha diseñado un temario preliminar para establecer un programa de formación accesible para los docentes del instituto siguiendo las recomendaciones de la Guía para Formación Accesible de los Involucrados en el Proceso de Enseñanza-aprendizaje en la Educación Superior, entregable 2.5 del proyecto "Asistencia tecnológica a la accesibilidad en la Educación Superior Virtual". La capacitación se basa en la sensibilización de los docentes ante la discapacidad y la accesibilidad (Bruder y Mogro-Wilson, 2010). La propuesta será revisada por las autoridades del instituto y se estructurará de acuerdo con los lineamientos de capacitación docente vigentes para oficializar su impartición. Se espera también que esta función de la unidad de atención a la accesibilidad digital se entrelace con otras iniciativas que el Tecnológico Nacional de México esta implementando a nivel nacional, como el Diplomado en Educación Inclusiva.

${ }^{1}$ URL:https://www.wgtn.ac.nz/disability/get-involved/disability-services-volunteers

Licencia Creative Commons Atribución 4.0 Internacional (CC BY 4.0)

Revista Cátedra, 5(1), pp. 106-118, enero-junio 2022. e-ISSN: 2631-2875

https://doi.org/10.29166/catedra.v5i1.3293 
- Movilidad académica para estudiantes con discapacidad (EN PLANIFICACIÓN), se espera colaborar estrechamente con el departamento de Gestión Tecnológica y Vinculación para formalizar convenios específicos para el aseguramiento de la movilidad de estudiantes con discapacidad, con la industria, el gobierno, y las fundaciones del Estado de Aguascalientes. Esta actividad se complementaría con el establecimiento de un proceso específico para la gestión de la movilidad accesible, el cual abarcará desde la difusión de información a los estudiantes, hasta la culminación de la estancia.

- Prácticas y estadías en la industria y fomento de la empleabilidad académica para estudiantes con discapacidad (EN PLANIFICACIÓN), de igual manera, se espera colaborar estrechamente con el departamento de Gestión Tecnológica y Vinculación para formalizar convenios específicos que garanticen la adecuada realización de prácticas y estadías en la industria; considerando incluso la posibilidad de la realización de prácticas laborales virtuales pudiendo utilizarse equipo propio del estudiante interesado, o bien las estaciones de trabajo accesibles de la unidad de atención a la accesibilidad digital. en este sentido, se visualiza también el desarrollo de simuladores laborales en 3D como una alternativa para el fomento de la empleabilidad de estudiantes con y sin discapacidad.

- Consultoría para docentes y personal (EN PLANIFICACIÓN), se visualiza que la unidad de atención a la accesibilidad digital esté disponible para asesorar a docentes del instituto que deseen implementar estrategias de accesibilidad en sus cursos (por ejemplo, adaptaciones curriculares), asimismo, se podría dar asesoría sobre tecnología accesible considerando los aspectos descritos en (Bruder \& MogroWilson, 2010).

- Desarrollar proyectos de investigación en el contexto de la asistencia tecnológica a la accesibilidad en la educación virtual (EN PLANIFICACIÓN), se visualiza el aprovechamiento de los equipos adquiridos a través del proyecto para realizar pruebas con usuarios de los diferentes desarrollos tecnológicos accesibles que realice la unidad de atención a la accesibilidad digital. Tanto los desarrollos como los resultados de sus pruebas serían estructurados como proyectos de investigación derivando en diversos tipos de publicaciones científicas dependiendo del impacto de los hallazgos obtenidos.

\subsection{La Unidad de Atención a la Accesibilidad Digital dentro de la estructura orgánica del TecNM/ITA}

Del mismo modo, los entregables del proyecto EduTech, mencionados al inicio del artículo, ayudaron a visualizar la organización interna de la unidad de atención y su adecuada integración a la estructura orgánica del TecNM/ITA. Las acciones en este contexto son descritas a continuación:

- Dependencia y operatividad de la unidad de atención a la accesibilidad digital (EN DESARROLLO), después de varias reuniones con las autoridades del instituto, se ha determinado que la unidad de atención tecnológica derivará de la Subdirección Académica (de la cual dependen todos los departamentos académicos y sus programas de estudio en el instituto) y se integrará a la Coordinación de Educación a Distancia. Actualmente se tienen ya identificados un par de espacios en el edificio de Educación a Distancia (cuya construcción finalizó en mayo de 2021), sin embargo, lo anterior aún está siendo formalizado por las autoridades del instituto. Del mismo modo, se considera hacer visible la unidad de atención como un servicio transversal en el website del instituto.

- Equipo de trabajo y colaboradores (EN DESARROLLO, el equipo de trabajo inicial integra a tres profesores con experiencia en las siguientes áreas de conocimiento: Profesor 1: Experiencia de usuarios, diseño centrado en las personas, Interacción Licencia Creative Commons Atribución 4.0 Internacional (CC BY 4.0) 
Humano-Computadora, tecnología educativa, y accesibilidad. Profesor 2: Diseño y desarrollo de videojuegos serios, Realidad Virtual, e Inteligencia Artificial. Profesor 3: Diseño y desarrollo web avanzados, y plataformas de educación virtual. Asimismo, se visualiza la integración de un profesor experto en Psicología y comportamiento humano. Este elemento se encuentra en proceso de formalización institucional. De manera inicial se consideran únicamente los perfiles mencionados anteriormente para el equipo de trabajo de la unidad. Sin embargo, se visualiza la participación externa de expertos en Lenguaje de Señas (Se espera tener reuniones con representantes del Sistema Nacional para el Desarrollo Integral de las Familias, DIF Municipal, en Aguascalientes para platicar sobre el proyecto) y Braille (Se ha identificado la Unión de Ciegos de Aguascalientes, vinculada a la Fundación ONCE Latino América con quien se espera tener reuniones para platicar sobre el proyecto).

- Aseguramiento de la calidad en la unidad de atención a la accesibilidad (EN PLANIFICACIÓN), se ha optado por implementar el conjunto de criterios sugeridos por The Quality Assurance Agency²; debido a que se trata de una estrategia confiable con un gran número de casos positivos de implementación reportados en la literatura; además, de ser compatible con múltiples escenarios de implementación. Sin embargo, es necesario que dicha alternativa sea corroborada por parte de las autoridades del instituto. Una vez formalizada la unidad de atención, se conformará la comisión de autoevaluación, considerando la participación de estudiantes con discapacidad. Se visualiza que la autoevaluación se realice de manera semestral. Se pretende realizar la primera autoevaluación una vez transcurrido el primer semestre con registro de atenciones y/o servicios otorgados por la unidad de atención. Se prevé la celebración de reuniones de análisis de los resultados de la autoevaluación al siguiente día hábil de la realización de esta.

\section{Conclusiones}

Este primer acercamiento al establecimiento de una Unidad de Atención a la Accesibilidad Tecnológica en el TecNM/ITA fue muy positivo y generó interés en el sistema nacional de tecnológicos. Del mismo modo, permitió corroborar la generalidad y fácil implementación de los hallazgos y recomendaciones provenientes de los entregables "1.1 Informe del estado de arte de unidades de atención a la accesibilidad tecnológica aplicada a la Educación Superior", y "2.1 Guía de implantación y procedimientos de gestión de una unidad de atención a la accesibilidad tecnológica aplicada a la Educación Superior" del proyecto EduTech. Por lo tanto, se puede inferir que podrá ayudar a otras instituciones de educación superior a conformar su propia unidad de atención a la accesibilidad tecnológica. En este sentido, y como parte del proyecto EduTech, se espera poder confirmar lo anterior mediante la reproducción del ejercicio en las demás universidades latinoamericanas socias del proyecto. Esta situación es importante, ya que aun cuando se tienen un avance considerable en la implementación de la unidad de atención, la mayoría de las acciones se encuentran en proceso de desarrollo e incluso, en proceso de planificación, impidiendo visualizar una implementación definitiva.

Por otro lado, el ejercicio también reveló algunos aspectos críticos a considerar para un resultado exitoso. El primero de ellos es sin duda el apoyo institucional; más allá del financiamiento proporcionado por la Unión Europea, fue necesario contar con la disposición de las autoridades del instituto para generar procesos específicos (por ejemplo, el proceso de licitación para la compra de equipos), asignar espacios para el montaje de la unidad de

2 https://nadp-uk.org/wp-content/uploads/2015/02/2010-Code-of-practice-for-academic-qualstandards.pdf 
atención a la accesibilidad tecnológica, y gestionar acciones específicas de varios departamentos y unidades del instituto (por ejemplo, recursos financieros, planeación y presupuestación, centro de cómputo, y servicios materiales). Otro elemento clave es el fomento de la participación de los profesores, y de los propios estudiantes con y sin discapacidad, en la estrategia planteada a través de la misión y visión de la unidad de atención a la accesibilidad tecnológica. Asimismo, se considera importante la vinculación con asociaciones externas, incluyendo a instancias de gobierno, específicamente enfocadas en la atención a las personas con discapacidad. 


\section{Agradecimientos}

Las acciones descritas en este artículo han sido cofinanciadas por el programa Erasmus+ de la Unión Europea EduTech (609785-EPP-1-2019-1-ES-EPPKA2-CBHE-JP). El apoyo de la Comisión Europea para la producción de esta publicación no constituye una aprobación del contenido, el cual refleja únicamente las opiniones de los autores, y la Comisión no se hace responsable del uso que pueda hacerse de la información contenida en la misma. / This paper has been co-funded by the Erasmus+ Programme of the European Union, project EduTech (609785-EPP-1-2019-1-ES-EPPKA2-CBHE-JP). The European Commission's support for the production of this publication does not constitute an endorsement of the contents, which reflect the views only of the authors, and the Commission cannot be held responsible for any use which may be made of the information contained therein.

\section{Bibliografía}

Alcantud, F., Ávila, V., y Asensi M. C. (2000). Integración de Estudiantes con Discapacidad en los Estudios Superiores. Universitat de València. Recuperado de https://oficinasuport.uib.cat/digitalAssets/108/108610 A4B3DF5Cd01.pdf

Bruder, M., y Mogro-Wilson, C. (2010). Student and faculty awareness and attitudes about students with disabilities. Review of Disability Studies: An International Journal, 6(2), 2-14.

Corredor Ponce, Z. A. (2016). Las adecuaciones curriculares como elemento clave para asegurar una educación inclusiva. Educación en Contexto, 2(3), 56-78. ISSN 24779296

Fundación Universia. (2017). Universidad y Discapacidad, III Estudio sobre el grado de inclusión del sistema universitario español respecto de la realidad de la discapacidad. Recuperado de http://riberdis.cedd.net/bitstream/handle/11181/5199/Universidad y discapaci dad III Estudio.pdf?sequence=1

Fundación Universia. (2018). Universidad y Discapacidad, IV Estudio sobre el grado de inclusión del sistema universitario español respecto de la realidad de la discapacidad. Recuperado de http://www.infocoponline.es/pdf/DISCAPACIDAD-UNIVERSIDAD.pdf

Ortego Hernando, J. L. (2000). Estudiantes con discapacidad y adaptaciones curriculares en los estudios superiores. Educació i Cultura, 13, 207-218.

Peralta, A., y Comunitaril, F. D. (2011). Libro Blanco sobre universidad y discapacidad. Recuperado de http://riberdis.cedd.net/bitstream/handle/11181/3015/165385.pdf?sequence=1 \&rd=003127559686103.

Porto-Castro, A. Mํ., y Gerpe-Pérez, E. Mํ. (2020). Servicios universitarios de atención al alumnado con discapacidad en España. Revista Española de Orientación y Psicopedagogía, 31(3), 149-169.

Sandoval, M., Morgado, B., y Doménech, A. (2020). University students with disabilities in Spain: faculty beliefs, practices and support in providing reasonable adjustments. Disability and Society, 36(5), 730-749.

TecNM. (2021). Breve historia de los Institutos Tecnológicos. Recuperado de https://www.tecnm.mx/?vista=Historia 
United Nations. (2006). Convention on the Rights of Persons with Disabilities (CRPD). Recuperado de

https://www.un.org/development/desa/disabilities/convention-on-the-rights-ofpersons-with-disabilities.html

\section{Autores}

RICARDO MENDOZA-GONZÁLEZ es profesor de tiempo completo en el Tecnológico Nacional de México / IT Aguascalientes. Miembro del Sistema Nacional de Investigadores (SNI-CONACYT, nivel I) 2017-2019. Doctor en Ciencias de la Computación (2009), y Maestro en Ciencias de la Computación (2008) por la Universidad Autónoma de Aguascalientes (UAA). Graduado de la Licenciatura en Informática por el Instituto Tecnológico de Aguascalientes (2004).

El Dr. Mendoza-González colabora activamente con profesores de Ontario Tech University (Canadá), Algoma University (Canadá), Østfold University College (Noruega), Universidad de Alcalá (España), Universidad de Alicante (España), Universidad Autónoma de Zacatecas (México) entre otras importantes Universidades y laboratorios como el UsaLab Laboratorio de Usabilidad de la Universidad Tecnológica de la Mixteca (Oaxaca, México). Asimismo, ha publicado su trabajo en prestigiosos foros internacionales, teniendo en su haber más de 60 publicaciones incluyendo tres libros y varios artículos en revistas indizadas, capítulos de libro, proceedings de conferencias internacionales y nacionales. El Dr. Mendoza-González tiene como principales temas de interés en investigación (más no limitados): Interacción Humano-Computadora, UX, User Research, Diseño Centrado en el Usuario, Accesibilidad tecnológica en la educación superior, Diseño de Interfaces para Dispositivos Móviles, Patrones de Diseño, Innovación tecnológica, Tecnologías Interactivas para la educación, MOOCs, Software Adaptativo, Interfaces Inteligentes, Algoritmos de Optimización, tecnología educativa, eHealth Communities, y Redes Neuronales.

JUAN-MIGUEL TOVAR-LUEVANO Egresado de Arquitectura en la Benemérita Universidad Autónoma de Aguascalientes en 1999, a partir de esa fecha inició en la construcción, en 2006 ingreso al Instituto Tecnológico de Aguascalientes adscrito al Departamento de Ciencias Básicas, jefe del Departamento de Planeación Programación y Presupuestación del 2012 a 2014, actualmente subdirector de Servicios Administrativos desde 2020 a la fecha. Miembro Numerario del Colegio de Arquitectos del Estado de Aguascalientes desde 2008.

Ha compartido sus actividades en la iniciativa privada, el servicio público y la docencia, permitiéndole una preparación continua y la oportunidad de participar en proyectos importantes para el TecNM / Instituto Tecnológico de Aguascalientes.

RAFAEL PORTILLO-ROSALES es Maestro en Ciencias de la Educación e Ingeniero en Sistemas Computacionales.

Actualmente subdirector Académico en el Instituto Tecnológico Nacional de México y director de Posgrados en Educación en la Universidad de Negocios y Estudios Profesionales UNEP. Interés de investigación en procesos de enseñanza y aprendizaje en ambientes virtuales, aprendizaje e inteligencia artificial, en procesos de certificación de competencias, y evaluación del aprendizaje. Áreas de conocimiento en diseño curricular, evaluación de aprendizaje, proceso de formación en entornos virtuales, diseño de recursos digitales y gestión de aprendizaje en plataformas virtuales. 